\title{
Meetings and Conferences
}

Visc Med 2017;33:483-485

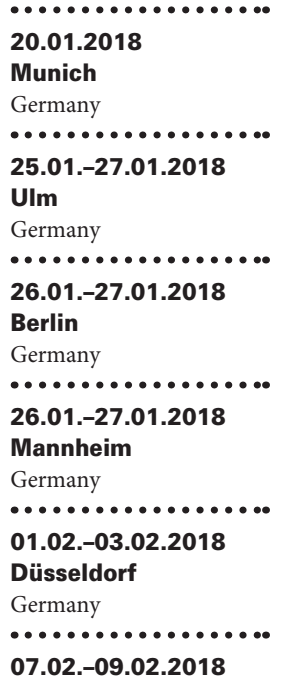

Kloster Drübeck

Germany

14.02.-17.02.2018

Vienna

Austria

(1)

17.02.-20.02.2018

Leipzig

Germany

21.02.-24.02.2018

Berlin

Germany

0000000000.000

23.02.2018

Hamburg

Germany

................

23.02.-24.02.2018

Nuremberg

Germany

…................

01.03.-03.03.2018

Neustadt/Weinstraße

Germany

...............

02.03.-03.03.2018

Freiburg i.Br.

Germany

[..................

02.03.-03.03.2018

Mannheim

Germany

•...............

02.03.-04.03.2018

Freising

Germany

.................

08.03.-10.03.2018

Mannheim

Germany
TZM-Essentials 2018

38. Jahrestagung des Deutschen Pankreasclubs

12. Jahrestagung der Gesellschaft für Gastroenterologie und Hepatologie in Berlin und Brandenburg

24. AGIO Winterkurs der DGVS

20. Internationales Endoskopie Symposium

DGHO Juniorakademie 2018

ECCO 2018 - European Cancer Congress

47. Jahrestagung der Deutschen Gesellschaft für Thorax-, Herz- und

Gefäßchirurgie

33. Deutscher Krebskongress

Endo Club Spring

Bundeskongress Chirurgie 2018

62. Jahrestagung der Saarländisch-Pfälzischen Internistischen

Gesellschaft

European Symposium on Minimally Invasive Surgery

Anorektaler Endosonographiekurs

25. Jahrestagung der Deutschen Gesellschaft für Neurogastroenterologie und Motilität

19. DGVS-Seminar Chronisch entzündliche Darmerkrankungen - Modul 1
Information: www.tzm-essentials.de

Information: www.dpc-kongress.de

Information: www.gghbb.de

\section{Information:}

www.dgvs.de/fortbildung-aktuell/dgvs-veranstaltungen

Information: www.endo-duesseldorf.com

Information: www.dgho-service.de/veranstaltungen/ dgho-juniorakademie-2018

Information: www.ecco-ibd.eu/eccols

Information: www.dgthg-jahrestagung.de

Information: www.dkk2018.de

Information: www.endoclubspring.de

Information: www.bundeskongress-chirurgie.de

Information: www.spig.org

Information: www.mis-symposium.de

Information: fuks@bkb.info

Information: www.neurogastro.de

Information:

www.dgvs.de/fortbildung-aktuell/dgvs-veranstaltungen

\section{KARGER}




\subsection{3.-17.03.2018}

Mannheim

Germany

...............

15.03.-17.03.2018

St. Gallen

Switzerland

..................

15.03.-17.03.2018

Munich

Germany

.................

15.03.-17.03.2018

Munich

Germany

14.04.-17.04.2018

Mannheim

Germany

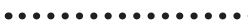

17.04.-20.04.2018

Berlin

Germany

20.04.-21.04.2018

Berlin

Germany

.................

27.04.-28.04.2018

Berlin

Germany

27.04.-28.04.2018

Berlin

Germany

......................

31.05.-02.06.2018

Leipzig

Germany

.................

06.06.-08.06.2018

Vienna

Austria

..................

07.06.-08.06.2018

Dortmund

Germany

.................

08.06.-09.06.2018

Ludwigshafen

Germany

09.06 .2018

Wiesbaden

Germany

•..............

14.06.-15.06.2018

Munich

Germany

..................

14.06.-16.06.2018

Salzburg

Austria

................

20.06.-23.06.2018

Cologne

Germany

21.06.-23.06.2018

Kassel

Germany

.................

29.06.-30.06.2018

Hanover

Germany
21. DGVS-Seminar Onkologische Gastroenterologie - Modul 1

4th International Gastrointestinal Cancer Conference - Primary Therapy of Early GI Cancer

48. Kongress der Deutschen Gesellschaft für Endoskopie und Bildgebende Verfahren

44. Deutscher Koloproktologen-Kongress

124. Kongress der Deutschen Gesellschaft für Innere Medizin (DGIM)

135. Kongress der Deutschen Gesellschaft für Chirurgie

12. AIO-GI-Tag

Endoskopie Live 2018

7. Hepatologie-Update-Seminar

0000000000000000000000000000000000000000000000000000000000

vereinigung und der Mitteldeutschen Gesellschaft für Gastroenterologie

59. Österreichischer Chirurgenkongress

Viszeralmedizin NRW 2018

6. Ludwigshafener GastroTage

14. Interdisziplinäres GI-Oncology Update

46. Jahrestagung der Gesellschaft für Gastroenterologie in Bayern

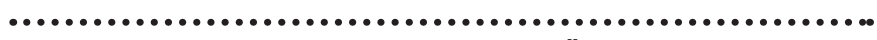

51. Jahrestagung und 29. Fortbildungskurs der Österreichischen

Gesellschaft für Gastroenterologie und Hepatologie

KIT 2018 - Kongress für Infektionskrankheiten und Tropenmedizin

Ernährung 2018 - Ernährung ist Therapie und Prävention

15. HepNet Symposium
Information:

www.dgvs.de/fortbildung-aktuell/dgvs-veranstaltungen

Information: www.oncoconferences.ch

Information: www.dge-bv.de

Information: www.koloproktologen-kongress.de

Information: www.dgim.de

Information: www.chirurgie2018.de

Information: aio@krebsgesellschaft.de

Information: www.endoskopie-live-berlin.de

Information: www.hepato-update.com

Information: www.mgfg.de

Information: www.chirurgenkongress.at

Information: www.viszeralmedizin-nrw.de

Information: www.gastrotagelu.de

Information: www.gi-oncology.de

Information: www.gfgb.org

Information: www.oeggh.at/veranstaltungen

Information: www.kit2018.de

Information: www.ernaehrung2018.de

Information:

www.deutsche-leberstiftung.de/symposium 
[................

30.06.2018

Wiesbaden

Germany

18.07.-20.07.2018

Garmisch-

Partenkirchen

Germany

•..............

12.09.-15.09.2018

Munich

Germany

.................

28.09.-02.10.2018

Vienna

Austria

-..............

02.11.-03.11.2018

Hamburg

Germany

..................

08.11.-10.11.2018

Mannheim

Germany

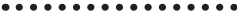

15.11.-17.11.2018

Mannheim

Germany

[.................

05.12.-08.12.2018

Berlin

Germany

-...................

06.03.-09.03.2019

Berlin

Germany
GI-Oncology - 14. Interdisziplinäres Update

Information: www.gi-oncology.de

95. Jahrestagung der Vereinigung Bayerischer Chirurgen (VBC) -

Ganzheitliche Chirurgie

Viszeralmedizin 2018

000000000000000000000000000000000000000000000000000000000000000

Jahrestagung der Deutschen, Österreichischen und Schweizerischen

Gesellschaften für Hämatologie und Medizinische Onkologie

Endo Club Nord 2018

21. DGVS-Seminar Onkologische Gastroenterologie - Modul 2

19. DGVS-Seminar Chronisch entzündliche Darmerkrankungen Modul 2

17. DGVS-Seminar Hepatologie - Modul 1

17. DGVS-Seminar Hepatologie - Modul 20
Information: www.www.vbc2018.de.de

Information: www.viszeralmedizin.com

Information: www.haematologie-onkologie-2018.com

Information: www.endoclubnord.de

\section{Information:}

www.dgvs.de/fortbildung-aktuell/dgvs-veranstaltungen

\section{Information:}

www.dgvs.de/fortbildung-aktuell/dgvs-veranstaltungen

\section{Information:}

www.dgvs.de/fortbildung-aktuell/dgvs-veranstaltungen

\section{Information:}

www.dgvs.de/fortbildung-aktuell/dgvs-veranstaltungen 